\title{
MRI Contrast Agents for Pharmacological Research
}

\author{
Enzo Terreno and Silvio Aime* \\ Department of Molecular Biotechnology and Health Sciences, Molecular and Preclinical Imaging Centers, University of \\ Torino, Torino, Italy
}

Keywords: molecular imaging, MRI, contrast agents, theranosis, pharmacology

\section{INTRODUCTION}

The advent of the molecular imaging era has offered to pharmacologists very powerful tools for drug discovery and development, in vivo evaluation of pharmacokinetic properties, and monitoring drug efficacy (Hargreaves, 2008; Nairne et al., 2015). In fact, molecular imaging technologies provide minimally invasive procedures to visualize, characterize, and quantify biological processes occurring at cellular/subcellular level (Weissleder and Mahmood, 2001), thus overcoming the poor clinical translatability often exhibited by in vitro/ex-vivo experimental models.

The continuous advances in biomedical imaging technologies may significantly boost the development of novel and more effective drugs, and accelerating the selection of lead compounds, with important time and costs benefits for healthcare.

In vivo imaging of drug delivery and release, as well as monitoring of the therapeutic outcomes, represent the base of personalized medicine, thus allowing patients to be successfully addressed to the more effective therapeutic regime.

Overall, the use of molecular imaging procedures aimed at supporting any therapeutic

Edited by:

Nicolau Beckmann,

Novartis Institutes for BioMedical

Research, Switzerland

Reviewed by:

Gustav Strijkers,

Academisch Medisch Centrum,

Netherlands

*Correspondence:

Silvio Aime

silvio.aime@unito.it

Specialty section:

This article was submitted to

Experimental Pharmacology and Drug

Discovery,

a section of the journal

Frontiers in Pharmacology

Received: 15 September 2015

Accepted: 19 November 2015

Published: 09 December 2015

Citation:

Terreno E and Aime S (2015) MRI Contrast Agents for Pharmacological

Research. Front. Pharmacol. 6:290.

doi: 10.3389/fphar.2015.00290

Focusing on pharmacological therapies, a typical theranostic procedure requires the design of an imaging-traceable agent, whose structure and properties are suitably tailored to the aims of the examination. Imaging drug-delivery allows the assessment of the accumulation of the drug at the biological target, thus helping the selection of the more appropriate treatment. To get accurate information, the imaging agent should have the same physico-chemical properties of the drug. This requirement can be successfully met by labeling pharmaceuticals (organic molecules, peptides, proteins, radiochelates) with PET- or SPECT-traceable radioisotopes, because of the minimal structural perturbation caused by the introduction of commonly used radionuclides (e.g., ${ }^{18} \mathrm{~F},{ }^{11} \mathrm{C},{ }^{123} \mathrm{I},{ }^{68} \mathrm{Ga},{ }^{111} \mathrm{In}$; Baum et al., 2010; Gains et al., 2011; Gomes et al., 2011; Witzig et al., 2013; Wynendaele et al., 2014).

On the other hand, when the drug is loaded into a nanocarrier, also the other available imaging modalities (CT, MRI, NIRF, US, PAI) can be used to visualize the delivery of the pharmaceutical. The imaging probe can be loaded in the carrier alone or together with the drug. The first option is preferable for drug selection, the second one for monitoring therapies. Among the imaging technologies, MRI is an excellent choice because combines exquisite spatial resolution, no limits in tissue penetration, and a vast portfolio of probes and contrast modalities that allows the design/selection of the best agent for any theranostic application.

\section{CLASSIFICATION OF MRI CONTRAST AGENTS}

MRI contrast agents can be grouped in five classes: $\mathrm{T}_{1}$ agents, $\mathrm{T}_{2} / \mathrm{T}_{2}{ }^{*}$ agents, CEST agents, ${ }^{19}$ F-based agents, and hyperpolarized probes (Figure 1). 


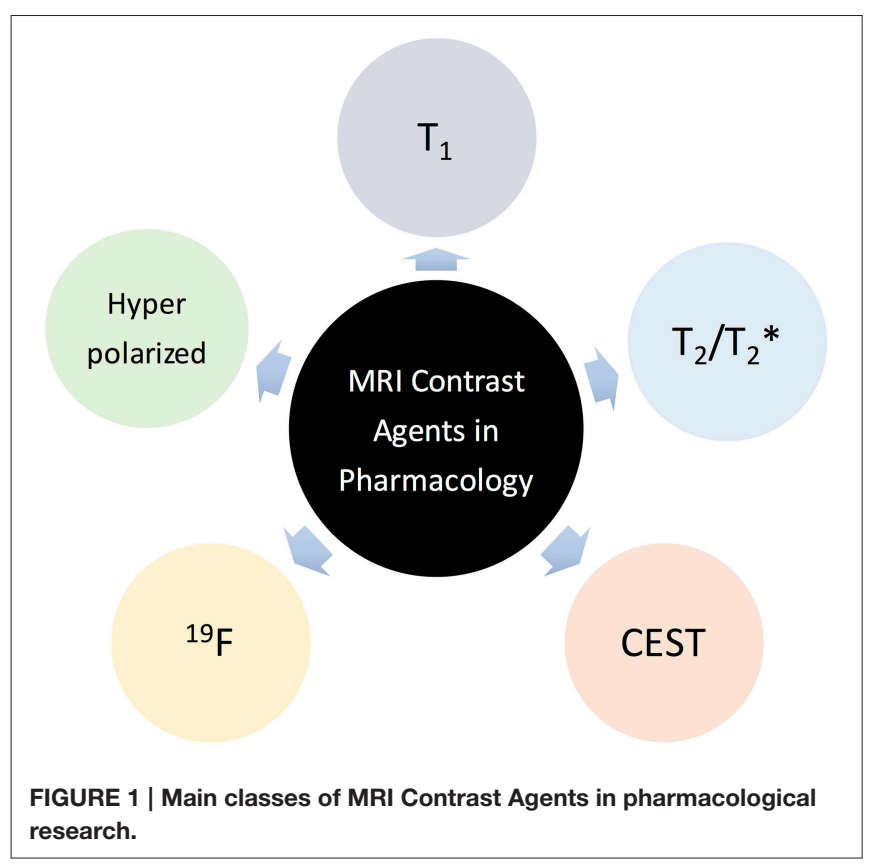

This section intends to provide the reader a brief description of these systems focusing on their main pro and cons with particular reference to the applications in pharmacological research.

\section{$T_{1}$ AGENTS}

$\mathrm{T}_{1}$ agents are mostly represented by paramagnetic metal [Gd(III) or $\mathrm{Mn}(\mathrm{II})]$ complexes that enhance the MR water signal (signal brightening) in $\mathrm{T}_{1 \mathrm{w}}$ scans. The main benefits using $\mathrm{T}_{1}$ agents relies on the high versatility of this contrast mechanism that is affected by a large number of factors related to either structural and dynamic characteristics of the agent or biological aspects like the intra-voxel distribution of the probe (e.g., intra/extravascular, intra/extra-cellular). A representative example is the use of paramagnetic complexes to visualize the delivery and the release of a drug from liposomes (Viglianti et al., 2006; Hijnen et al., 2014; Rizzitelli et al., 2015). However, such systems displayed a limited sensitivity that makes necessary a local concentration of agent around $10 \mu \mathrm{M}$. This drawback can be partially overcome using nanometric materials that can aggregate even millions of contrastographic units, thus reducing significantly the local concentration of contrast agent (here represented by the nanosystem) necessary to generate a detectable $\mathrm{T}_{1}$ contrast.

\section{$T_{2} / T_{2}{ }^{*}$ AGENTS}

$\mathrm{T}_{2} / T_{2}{ }^{*}$ agents are chemicals, mostly superparamagnetic nanoparticles made of iron oxides, capable to shorten the $T_{2} / T_{2}{ }^{*}$ of water protons much more than $\mathrm{T}_{1}$. Thus, their presence in the MRI image is signaled by a signal loss (darkening). Such nanoparticles show an intrinsic higher sensitivity than $\mathrm{T}_{1}$ agents that justifies their extensive use in MR-molecular imaging procedures, especially for cellular imaging (Srivastava et al., 2015). On the other side, these agents are often considered not to be the candidates of choice for designing smart agents, due to the difficulty to modulate the $\mathrm{T}_{2} / \mathrm{T}_{2}{ }^{*}$ contrast as a function of the microenvironment characteristics. Furthermore, the signal loss is not desirable when the target site has an intrinsically low signal (e.g., lungs, hemorrhages). However, interesting and promising theranostic applications of iron oxide nanoparticles for the visualization of drug delivery and release have been recently published (Krol et al., 2013; Liu et al., 2013).

\section{CEST AGENTS}

The family of CEST agents is constantly growing, and though there are no agents in clinical trials yet, the peculiarities of these systems could open new and interesting future perspectives for MRI agents in pharmacology research. The acronym CEST stands for Chemical Exchange Saturation Transfer and identifies those chemicals that generate a MRI contrast through the transfer, mediated by chemical exchange, of saturated (i.e., irradiated with a frequency specific RF pulse) protons from the donor pool (CEST agent) to the acceptor pool (bulk water).

The most important advantage of using CEST agents is that the contrast can be detected only following the irradiation of the specific NMR resonance of the donor pool. It follows that the detection of the agent is frequency-encoded and this property can be exploited for multiplex imaging or for the design of concentration-independent smart agents, both tasks being very challenging in the case of the above described relaxation agents.

The sensitivity of CEST contrast detection was recognized as an issue since the early days of the development of these agents (Ward et al., 2000). Few mM of the donor pool are necessary. However, in analogy with $\mathrm{T}_{1}$ agents, a large sensitivity gain can be accomplished by recurring to nanosystems. As far as the use of CEST agents in pharmacology, excellent results have been obtained using liposomes as carriers of a huge amount of exchanging water protons (the water molecules entrapped in the nanovesicles) properly shifted by the entrapment of a paramagnetic shift reagent in the inner aqueous compartment. The resulting systems (called LipoCEST, Aime et al., 2005) have been demonstrated to be very promising for imaging drug release at preclinical level (Langereis et al., 2009; Delli Castelli et al., 2010; Castelli et al., 2014).

\section{HETERONUCLEAR AGENTS}

CEST agents share the frequency-encoded contrast property with agents containing MRI detectable nuclei different from protons. Among them, two classes deserve to be mentioned here because there are compounds already approved for humans or in advanced clinical trials: ${ }^{19} \mathrm{~F}$ agents and hyperpolarized probes.

\section{${ }^{19} \mathrm{~F}$ AGENTS}

${ }^{19} \mathrm{~F}$ nuclei are the most sensitive spins after protons, and, therefore can be detected by MRI without any enrichment. The 
detection sensitivity is similar to CEST agents (few mM of fluorine atoms). Consequently, ${ }^{19} \mathrm{~F}$ agents are almost exclusively represented by nanosystems, among which perfluorocarbon nanoparticles (PFCs) are by far the most commonly used (Jacoby et al., 2013). The important advantage of fluorinated agents over the other class of contrast media stems from the possibility to directly correlate the MR signal to the agent concentration, thus allowing the quantification of targeted biomarkers and/or drugs delivered at the site of interest (Lanza et al., 2002). A commercially available formulation of PFCs will enter soon in clinical phase 1 for labeling and in vivo tracking human adiposederived stem cells for breast reconstruction $\left[{ }^{19} \mathrm{~F}\right.$ Hot Spot MRI of Human Adipose-derived Stem Cells for Breast Reconstruction (CS-1000), ID NCT02035085, source: ClinicalTrials.gov].

\section{HYPERPOLARIZED PROBES}

This class of MRI agents is by far the most sensitive one, owing to the use of polarization techniques (like dynamic nuclear polarization, DNP, laser optical pumping, para-hydrogen induced polarization) that increase dramatically (up to five order of magnitude) the population difference between the spin energy levels. These agents have some similarity with PET tracer, not only for the excellent sensitivity, but also for the decay of the signal they generate (caused by the return back to the thermal polarization) that occurs on the timescale of the $T_{1}$ of the polarized spin. Hence, one limitation in the use of hyperpolarized probes is the signal loss over time that requires fast injection and rapid accumulation at the target site.

Hyperpolarized gases (e.g., ${ }^{3} \mathrm{He}$ and ${ }^{129} \mathrm{Xe}$ ) are clinically used for imaging the respiratory apparatus (Liu et al., 2014), whereas a ${ }^{13} \mathrm{C}$ hyperpolarized compound $\left({ }^{13} \mathrm{C}\right.$ pyruvate) is currently in phase 1 clinical trial as metabolic agents for prostate cancer diagnosis (University of California, 2010). Besides cancer, 13C hyperpolarized agents are under intense scrutiny in cardiovascular research (Rider and Tyler, 2013).

The use of hyperpolarized probes for imaging drug delivery is quite limited, mainly due to the time constrain. Hence, their

\section{REFERENCES}

Aime, S., Delli Castelli, D., and Terreno, E. (2005). Highly sensitive MRI chemical exchange saturation transfer agents using liposomes. Angew. Chem. Int. Ed. Engl. 44, 5513-5515. doi: 10.1002/anie.200501473

Baum, R. P., Prasad, V., Müller, D., Schuchardt, C., Orlova, A., Wennborg, A., et al. (2010). Molecular imaging of HER2-expressing malignant tumors in breast cancer patients using synthetic $111 \mathrm{In}$ - or $68 \mathrm{Ga}$-labeled affibody molecules. J. Nucl. Med. 51, 892-897. doi: 10.2967/jnumed.109.073239

Castelli, D. D., Boffa, C., Giustetto, P., Terreno, E., and Aime, S. (2014). Design and testing of paramagnetic liposome-based CEST agents for MRI visualization of payload release on $\mathrm{pH}$-induced and ultrasound stimulation. J. Biol. Inorg. Chem. 19, 207-214. doi: 10.1007/s00775-013-1042-0

Delli Castelli, D., Dastrù, W., Terreno, E., Cittadino, E., Mainini, F., Torres, E., et al. (2010). In vivo MRI multicontrast kinetic analysis of the uptake and intracellular trafficking of paramagnetically labeled liposomes. J. Control. Release 144, 271-279. doi: 10.1016/j.jconrel.2010.03.005 impact in pharmacological research is primarily in monitoring therapy outcome (Laustsen et al., 2014; Park et al., 2014).

A very intriguing combination between hyperpolarized and CEST agents has been proposed using ${ }^{129} \mathrm{Xe}$-based probes. The contrast arising from these agents (dubbed Hyper-CEST) relies on the reversible binding of hyperpolarized Xe with a macrocyclic host (e.g., cryptophane, cucurbituril; Schröder et al., 2006). The large chemical shift difference between the exchanging free and host-bound species allows the generation of a CEST contrast where the presence of very small amounts of the host-bound Xe can be detected after transferring its saturation to the signal of the free gas. In vitro proof-of-concepts highlighting the great potential and high sensitivity of these agents has been very recently published (Kunth et al., 2015; Schnurr et al., 2015).

\section{CONCLUSIONS}

In spite of the intrinsic limited sensitivity of NMR/MRI response, several routes have been identified to allow the use of MRI probes in pharmacological studies. The enhanced sensitivity allows to take advantage of the superb spatial and temporal resolution of the MR imaging modality. On this basis, MRI is increasing its competitiveness in the Molecular Imaging arena, allowing the design of innovative experiments that provide a detailed picture of the biological microenvironment at cellular and molecular level. Moreover, functional and molecular MRI investigations imply a level of invasiveness that is definitively low in respect to the commonly used probes for nuclear medicine.

Finally, the use of frequency-encoding agents opens new horizons as they allow the visualization of more targets in the same anatomical region, i.e., they provide the access to multicolor MR images of the kind the biomedical operators are used to deal with in the histological characterization of bioptical specimens.

\section{AUTHOR CONTRIBUTIONS}

Both the authors contributed to: (i) the design and organization of the manuscript, (ii) the drafting and critical revising of the article, and (iii) the approval of the final version to be published.

Gains, J. E., Bomanji, J. B., Fersht, N. L., Sullivan, T., D’Souza, D., Sullivan, K. P., et al. (2011). 177Lu-DOTATATE Molecular Radiotherapy for Childhood Neuroblastoma. J. Nucl. Med. 52, 1041-1047. doi: 10.2967/jnumed.110.085100

Gomes, C. M., Abrunhosa, A. J., Ramos, P., and Pauwels, E. K. (2011). Molecular imaging with SPECT as a tool for drug development. Adv. Drug Deliv. Rev. 63, 547-554. doi: 10.1016/j.addr.2010.09.015

Hargreaves, R. J. (2008). The role of molecular imaging in drug discovery and development. Clin. Pharmacol. Ther. 83, 349-353. doi: 10.1038/sj.clpt.6100467

Hijnen, N. M., Langereis, S., and Grüll, H. (2014). Magnetic resonance guided high-intensity focused ultrasound for image-guided temperature-induced drug delivery. Adv. Drug Deliv. Rev. 72, 65-81. doi: 10.1016/j.addr.2014.01.006

Jacoby, C., Temme, S., Mayenfels, F., Benoit, N., Krafft, M. P., Schubert, R., et al. (2013). Probing different perfluorocarbons for in vivo inflammation imaging by 19F MRI: image reconstruction, biological half-lives and sensitivity. NMR Biomed. 27, 261-271. doi: 10.1002/nbm.3059

Krol, S., Macrez, R., Docagne, F., Defer, G., Laurent, S., Rahman, M., et al. (2013). Therapeutic benefits from nanoparticles: the potential significance of 
nanoscience in diseases with compromise to the blood brain barrier. Chem. Rev. 113, 1877-1903. doi: 10.1021/cr200472g

Kunth, M., Witte, C., Hennig, A., and Schroder, L. (2015). Identification, classification, and signal amplification capabilities of high-turnover gas binding hosts in ultra-sensitive NMR. Chem. Sci. 6, 6069-6075. doi: 10.1039/C5SC01400J

Lammers, T., Aime, S., Hennink, W. E., Storm, G., and Kiessling, F. (2011). Theranostic nanomedicine. Acc. Chem. Res. 44, 1029-1038. doi: $10.1021 /$ ar200019c

Langereis, S., Keupp, J., van Velthoven, J. L., de Roos, I. H., Burdinski, D., Pikkemaat, J. A., et al. (2009). A temperature-sensitive liposomal 1H CEST and 19F contrast agent for MR image-guided drug delivery. J. Am. Chem. Soc. 131, 1380-1381. doi: 10.1021/ja8087532

Lanza, G. M., Yu, X., Winter, P. M., Abendschein, D. R., Karukstis, K. K., Scott, M. J., Chinen, L. K., et al. (2002). Targeted antiproliferative drug delivery to vascular smooth muscle cells with a magnetic resonance imaging nanoparticle contrast agent: implications for rational therapy of restenosis. Circulation 106, 2842-2847. doi: 10.1161/01.CIR.0000044020.27990.32

Laustsen, C., Lipsø, K., Ostergaard, J. A., Nørregaard, R., Flyvbjerg, A., Pedersen, M., et al. (2014). Insufficient insulin administration to diabetic rats increases substrate utilization and maintains lactate production in the kidney. Physiol. Rep. 11:e12233. doi: 10.14814/phy2.12233

Liu, J., Detrembleur, C., Debuigne, A., De Pauw-Gillet, M. C., Mornet, S., Vander Elst, L., et al. (2013). Poly(acrylic acid)-block-poly(vinyl alcohol) anchored maghemite nanoparticles designed for multi-stimuli triggered drug release. Nanoscale 5, 11464-11477. doi: 10.1039/c3nr02861e

Liu, Z., Araki, T., Okajima, Y., Albert, M., and Hatabu, H. (2014). Pulmonary hyperpolarized noble gas MRI: recent advances and perspectives in clinical application. Eur. J. Radiol. 83, 1282-1291. doi: 10.1016/j.ejrad.2014. 04.014

Nairne, J., Iveson, P. B., and Meijer, A. (2015). Imaging in drug development. Prog. Med. Chem. 54, 231-280. doi: 10.1016/bs.pmch.2014.10.002

Park, I., Mukherjee, J., Ito, M., Chaumeil, M. M., Jalbert, L. E., Gaensler, K., et al. (2014). Changes in pyruvate metabolism detected by magnetic resonance imaging are linked to DNA damage and serve as a sensor of temozolomide response in glioblastoma cells. Cancer Res. 74, 7115-7124. doi: 10.1158/00085472.CAN-14-0849

Rider, O. J., and Tyler, D. J. (2013). Clinical implications of cardiac hyperpolarized magnetic resonance imaging. J. Cardiovasc. Magn. Reson. 15:93. doi: 10.1186/1532-429X-15-93

Rizzitelli, S., Giustetto, P., Cutrin, J. C., Delli Castelli, D., Boffa, C., Ruzza, M., et al. (2015). Sonosensitive theranostic liposomes for preclinical in vivo MRI-guided visualization of doxorubicin release stimulated by pulsed low intensity non-focused ultrasound. J. Control. Release 202, 21-30. doi: 10.1016/j.jconrel.2015.01.028

Schnurr, M., Sloniec-Myszk, J., Döpfert, J., Schröder, L., and Hennig, A. (2015). Supramolecular assays for mapping enzyme activity by displacement-triggered change in hyperpolarized (129)Xe magnetization transfer NMR spectroscopy. Angew. Chem. Int. Ed. Engl. 54, 13444-13447. doi: 10.1002/anie.201507002

Schröder, L., Lowery, T. J., Hilty, C., Wemmer, D. E., and Pines, A. (2006). Molecular imaging using a targeted magnetic resonance hyperpolarized biosensor. Science 314, 446-449. doi: 10.1126/science.1131847

Srivastava, A. K., Kadayakkara, D. K., Bar-Shir, A., Gilad, A. A., McMahon, M. T., and Bulte, J. W. (2015). Advances in using MRI probes and sensors for in vivo cell tracking as applied to regenerative medicine. Dis. Model Mech. 8, 323-336. doi: 10.1242/dmm.018499

University of California (2010). Hyperpolarized Pyruvate Injection in Subjects with Prostate Cancer, Phase 1 Clinical Trial, ID NCT01229618. Available online at: clinicaltrials.gov

Viglianti, B. L., Ponce, A. M., Michelich, C. R., Yu, D., Abraham, S. A., Sanders, L., et al. (2006). Chemodosimetry of in vivo tumor liposomal drug concentration using MRI. Magn. Reson. Med. 56, 1011-1018. doi: 10.1002/mrm.21032

Ward, K. M., Aletras, A. H., and Balaban, R. S. (2000). A new class of contrast agents for MRI based on proton chemical exchange dependent saturation transfer (CEST). J. Magn. Reson. 143, 79-87. doi: 10.1006/jmre.1999.1956

Weissleder, R., and Mahmood, U. (2001). Molecular imaging. Radiology 219, 316-333. doi: 10.1148/radiology.219.2.r01ma19316

Witzig, T. E., Wiseman, G. A., Maurer, M. J., Habermann, T. M., Micallef, I. N., Nowakowski, G. S., et al. (2013). A phase I trial of immunostimulatory CpG 7909 oligodeoxynucleotide and 90-yttrium ibritumomab tiuxetan radioimmunotherapy for relapsed B-cell non-Hodgkin lymphoma. Am. J. Hematol. 88, 589-593. doi: 10.1002/ajh.23460

Wynendaele, E., Bracke, N., Stalmans, S., and De Spiegeleer, B. (2014). Development of peptide and protein based radiopharmaceuticals. Curr. Pharm. Des. 20, 2250-2267. doi: 10.2174/13816128113196660663

Conflict of Interest Statement: The authors declare that the research was conducted in the absence of any commercial or financial relationships that could be construed as a potential conflict of interest.

Copyright (c) 2015 Terreno and Aime. This is an open-access article distributed under the terms of the Creative Commons Attribution License (CC BY). The use, distribution or reproduction in other forums is permitted, provided the original author(s) or licensor are credited and that the original publication in this journal is cited, in accordance with accepted academic practice. No use, distribution or reproduction is permitted which does not comply with these terms. 